neonatal and pediatric intensive care units. Am J Infect Control 2001;29:152-157.

Girouard S, Levine G, Goodrich K, Jones S, Keyserling H, Rathore M, et al. Pediatric Prevention Network: a multicenter collaboration to improve health care outcomes. Am J Infect Control 2001;29:158-161.

\section{Antibiotic-Resistant Bacteria Among Long-Term-Care Facility Residents}

There is limited information on antibiotic-resistant bacteria in community long-term-care facilities (LTCFs). Mylotte and coinvestigators have reported on a study whose objective was to obtain data on resistant organisms in residents from community LTCFs admitted to an inpatient acute geriatrics service (AGS). Two studies were performed. In the first study, bacteriology records of all admissions to the AGS for the period from November 1, 1998 , through June 30,2000 , were reviewed for resistant organisms (methicillin-resistant Staphylococcus aureus [MRSA], vancomycin-resistant enterococci [VRE], and resistant gram-negative bacilli). In the second study, residents admitted to the AGS during a 2-month period ( $\mathrm{n}=92$ admissions) had surveillance cultures (nares, gastrostomy site, wounds, and urine) for resistant organisms performed within 72 hours of admission.

In the retrospective study, there were 727 admissions, of which $437(60 \%)$ had 928 cultures within 72 hours of admission; $590(64 \%)$ cultures grew one or more pathogens. Urine (65\%) and blood (26\%) cultures accounted for $91 \%$ of all cultures done. Rates of resistance by culture site were as follows: urine (resistant organism in 16.6\% of 373 cultures), blood ( $6.7 \%$ of 60 cultures), wound (52\% of 23 cultures), and sputum ( $40 \%$ of 20 cultures). MRSA and enterococci with high-level gentamicin resistance were the most common resistant organisms identified. No VRE were isolated; only $3 \%$ of $421 \mathrm{gram}$-negative isolates were considered resistant strains, compared with $19 \%(P<.001)$ of grampositive isolates. In the prospective study, $17 \%$ of 92 residents were found to have a resistant organism in 1 or more surveillance cultures; the most common resistant organisms were MRSA and high-level gentamicin-resistant enterococci. Only 1 resident was found to have VRE in a rectal swab culture; resistant gram-negative bacilli also were uncommon.

The authors concluded that, among residents of community LTCFs admitted to an AGS, resistant organisms were identified infrequently ( $<20 \%$ of admissions). MRSA was the most common resistant organism; VRE and resistant gram-negative bacilli were rare. These findings vary from other studies suggesting that there may be geographic variation in the epidemiology of resistant organisms among residents of community LTCFs.

FROM: Mylotte JM, Goodnough S, Tayara A. Antibiotic-resistant organisms among long-term care facility residents on admission to an inpatient geriatrics unit: retrospective and prospective surveillance. Am J Infect Control 2001;29:139-144.

\section{MRSA in Latin America: 1996 to 1998}

Aires De Sousa and colleagues from the Universidade Nova de Lisboa, Oeiras, Portugal, studied 493 methicillinresistant Staphylococcus aureus (MRSA) isolates recovered from 1996 to 1998 from 22 hospitals in five countries of Latin America: Argentina, Brazil, Chile, Uruguay, and Mexico. The isolates were examined for antimicrobial susceptibility and clonal type so as to define the endemic clones in those hospitals. The hybridization of $\mathrm{ClaI}$ restriction digests with the mecA- and Tn554-specific DNA probes combined with pulsed-field gel electrophoresis of chromosomal SmaI digests (ClaI-mecA::ClaI-Tn554::PFGE clonal types) documented not only the predominance and persistence of the Brazilian clone (XI::B::B) in Brazil (97\%) and Argentina $(86 \%)$ but also its massive dissemination to Uruguay (100\%). Moreover, a close relative of the Brazilian clone (XI::kappa::B) was highly represented in Chile (53\%), together with a novel clone (47\%) (II:: $\left.\mathrm{E}^{\prime}:: \mathrm{F}\right)$ resistant to penicillin, oxacillin, ciprofloxacin, chloramphenicol, clindamycin, erythromycin, and gentamicin. A unique clonal type (I::NH::M) was detected in Mexico among pediatric isolates and was resistant to penicillin, oxacillin, and gentamicin only.

This study clearly documented the very large capacity for geographic expansion and the persistence of the Brazilian clone, contributing not only to the increasing uniformity of the MRSA in South America but worldwide as well.

FROM: Aires De Sousa M, Miragaia M, Santos Sanches I, Avila S, Adamson I, Casagrande ST, et al. Threeyear assessment of methicillin-resistant Staphylococcus aureus clones in Latin America from 1996 to 1998. J Clin Microbiol 2001;39:2197-2205.

\section{Povidone-Iodine Versus Chlorhexidine Gluconate-Impregnated Dressing for Prevention of CVC Infections in Neonates}

Neonates who require a central venous catheter (CVC) for prolonged vascular access experience high rates of catheter-related (CR) bloodstream infection (BSI). Garland and coinvestigators conducted a multicenter, randomized clinical trial to ascertain the efficacy of a novel chlorhexidine-impregnated dressing (Biopatch Antimicrobial Dressing; Johnson \& Johnson Medical, Arlington, TX) on the CVC sites of neonates for the prevention of catheter-tip colonization, CR BSI, and BSI without a source. The trial was conducted in six level III neonatal intensive care units. Patients studied were neonates admitted to study units who would require a CVC for at least 48 hours. Eligible infants were randomized before catheter placement to 1 of the 2 catheter-site antisepsis regimens: (1) $10 \%$ povidone-iodine (PI) skin scrub, or (2) a $70 \%$ alcohol scrub followed by placement of a chlorhexidineimpregnated disk over the catheter insertion site. A transparent polyurethane dressing (Bioclusive Transparent 
Dressing; Johnson \& Johnson) was used to cover the insertion site in both study groups. Primary study outcomes evaluated were catheter-tip colonization, CR BSI, and BSI without an identified source.

Seven hundred five neonates were enrolled in the trial; 335 were randomized to receive the chlorhexidine dressing and 370 to skin disinfection with PI (controls). Neonates randomized to the antimicrobial dressing group were less likely to have colonized CVC tips than control neonates (15.0\% vs $24.0 \%$; relative risk [RR], $0.6 ; 95 \%$ confidence interval $\left[\mathrm{CI}_{95}\right.$, $0.5-0.9)$. Rates of CR BSI (3.8\% vs 3.2\%; RR, $1.2 ; \mathrm{CI}_{95}, 0.5-2.7$ ) and BSI without a source (15.2\% vs $14.3 \%$; RR, $1.1 ; \mathrm{CI}_{95}, 0.8-$ 1.5) did not differ between the two groups. Localized contact dermatitis from the antimicrobial dressing, requiring crossover into the PI treatment group, occurred in 15 (15.3\%) of 98 exposed neonates weighing $\leqslant 1,000 \mathrm{~g}$. No neonates in the PI group developed contact dermatitis.

The authors concluded that the novel chlorhexidineimpregnated dressing, replaced weekly, was as effective as cutaneous disinfection with $10 \%$ PI and redressing the site every 3 to 7 days for preventing CR BSI and BSI without a source in critically ill neonates requiring prolonged central venous access. The risk of local contact dermatitis under the chlorhexidine dressing limits its use in low-birthweight infants who require prolonged central access during the first 2 weeks of life.

FROM: Garland JS, Alex CP, Mueller CD, Otten D, Shivpuri C, Harris MC, et al. A randomized trial comparing povidone-iodine to a chlorhexidine gluconate-impregnated dressing for prevention of central venous catheter infections in neonates. Pediatrics 2001;107:1431-1436.

\section{Virucidal Activity of Ortho-Phthalaldehyde Solution Against Duck Hepatitis B Virus}

At the recent APIC 2001 meetings in Seattle, Roberts and Chan-Myers reported on studies where orthophthalaldehyde, a new high-level disinfectant alternative to glutaraldehyde, was tested for efficacy against duck hepatitis $B$ virus. Duck hepatitis B virus is accepted by the EPA as the surrogate test virus for human hepatitis B virus. Dilute $(0.3 \%)$ ortho-phthalaldehyde solutions were used in inactivation studies against duck hepatitis $B$ virus contained in whole duck serum. The virus was dried onto the bottom of petri dishes, and a quantity of dilute ortho-phthalaldehyde solution was added. After an exposure of 5 minutes at $20^{\circ} \mathrm{C}$, the virus was recovered and titrated on monolayers of duck hepatocytes.

After incubation there was no viable duck hepatitis B virus detected. In conclusion, the findings of this study demonstrate that ortho-phthalaldehyde solutions are efficacious against duck hepatitis $B$ virus at a 5-minute exposure at $20^{\circ} \mathrm{C}$.
FROM: Roberts C, Chan-Myers HB. Virucidal activity of ortho-phthalaldehyde solution against duck hepatitis B virus. Presented at the 28th APIC Annual Educational Conference and International Meeting; June 10-14, 2001; Seattle, WA.

\section{Hepatitis B and C Viruses: Where Are Germicides Relevant?}

Sattar and colleagues from the Centre for Research on Environmental Microbiology, Faculty of Medicine, University of Ottawa, and the Division of Bloodborne Pathogens, Health Canada, Ottawa, have published an upto-date primer on hepatitis B virus (HBV) and hepatitis C virus (HCV). Both viruses are the most prevalent bloodborne pathogens. Infections caused by these organisms can become chronic and may lead to liver cirrhosis and carcinoma. Limited chemotherapy is now available, but only $\mathrm{HBV}$ can be prevented through vaccination. Both viruses are enveloped and relatively sensitive to many physical and chemical agents; their ability to survive in the environment may not be as high as often believed. As a result, their spread occurs mainly through direct parenteral or percutaneous exposure to tainted body fluids and tissues. Careful screening of, and avoiding contact with, such materials remain the most effective means of protection. Nevertheless, the indirect spread of these viruses, although much less common, can occur when objects that are freshly contaminated with tainted blood enter the body or contact damaged skin.

Germicidal chemicals are important in the prevention of HBV and HCV spread through shared injection devices, sharps used in personal services (such as tattooing and body piercing), heat-sensitive medical or dental devices (such as flexible endoscopes), and in the cleanup of blood spills. Microbicides in vaginal gels also may interrupt their transmission. General-purpose environmental disinfection is unlikely to play a significant role in the prevention of the transmission of these viruses. Testing of low-level disinfectants and label claims for such products against HBV and $\mathrm{HCV}$ should be discouraged. Both viruses remain difficult to work with in the laboratory, but closely related animal viruses (such as the duck HBV) and the bovine viral diarrhea virus show considerable promise as surrogates for $\mathrm{HBV}$ and HCV, respectively. Although progress in the culturing of $\mathrm{HBV}$ and $\mathrm{HCV}$ is still underway, critical issues on virus survival and inactivation should be addressed with the use of these surrogates.

FROM: Sattar SA, Tetro J, Springthorpe VS, Giulivi A. Preventing the spread of hepatitis $B$ and $C$ viruses: Where are germicides relevant? Am J Infect Control 2001;29:187-197. 